\title{
Design of Ship Course-Keeping Autopilot using a Sine Function-Based Nonlinear Feedback Technique
}

\author{
Xian-ku Zhang and Guo-qing Zhang \\ (Navigation College, Dalian Maritime University (DMU), Liaoning 116026 China) \\ (E-mail: zgq_dlmu@163.com)
}

\begin{abstract}
Course keeping for ships is the core of automatic navigation in sea transportation. Much work has concentrated on developing novel control strategies for closed loop systems. We have turned our attention the other way to improve the control performance of marine autopilots in this work by "modulating" the control error using a sine function while the construction of the controller is not changed. The nonlinear feedback signal thus obtained is sent to the controller to replace the control error itself, which used to be the deviation between the output response and the reference input of the system. Such a control scheme is called "nonlinear feedback control" hereafter. Theoretical analysis by using a describing function and robust control theory shows that the same control quality is guaranteed with minor control actions for the nonlinear feedback scheme. Simulation experiments were carried out for the ship Yulong of Dalian Maritime University. It is shown that the method postulated in this paper has advantages of safety and energy saving in navigation; the maximum initial rudder angle is reduced by $31 \cdot 2 \%$ with satisfactory control effect.
\end{abstract}

\section{KEYWORDS}
1. Navigation.
2. Closed-loop gain shaping.
5. Sine function.
3. Course keeping.
4. Nonlinear feedback.
6. Describing function.

Submitted: 16 September 2014. Accepted: 24 July 2015. First published online: 2 September 2015.

1. INTRODUCTION. In recent years, research into linear feedback control has been richly developed and matured. To compensate the nonlinearity existing in the real plant, nonlinear control provides a unified approach for control design such as the Backstepping method, exact feedback linearisation, etc (Krstic et al., 1995). The value of these strategies is verified by the fact that many papers in the field are published. However, few works about nonlinear feedback control are apparent through searching the Web of Knowledge. In ship motion control, a small rudder amplitude and slow rudder ratio mean energy saving and abrasion reduction in the steering motor during manoeuvring. In heavy sea states, steering at large rudder angles can bring about increased rolling amplitudes, causing dangerous navigation situations (Johansen and Fossen, 2013). Therefore, the initial rudder angle and the steering 
frequency are required to be as small as possible when the control algorithm of autopilot for ships is designed (Aarsaether and Moan, 2010).

Course keeping control for ships can be taken as a benchmark problem in the study of ship motion control (Fossen, 2011). It is used as a test bed to demonstrate the control effect when a new control algorithm is developed. In Du et al. (2007), an adaptive course controller for time-varying parametrically uncertain nonlinear ships with completely unknown time-varying bounded control coefficients was developed and the design method did not require any a priori knowledge of the sign of the unknown time-varying control coefficient. Unlike the existing works (Bian et al., 2009; Ho et al., 2010), the heading autopilot in Velasco et al. (2013) was investigated based on an autonomous In-Scale Fast Ferry. The physical control system was implanted by a PC using WiFi communications, and the results are very valuable for coursekeeping autopilot design. Satpati et al. (2008) presented the design of a robust course controller for a cargo ship interacting with an uncertain environment using Particle Swarm Optimisation (PSO) -enabled automated Quantitative Feedback Theory (QFT). The plant dynamics were described as a second order Nomoto model with structure parametric variation. Simulation experiments showed the validity of the algorithm. A ship course-keeping control scheme of Robust Least Squares Support Vector Machine (RLSSVM) was proposed in Liu et al. (2010), which made full use of the nonlinear mapping ability, self-learning adaptability and parallel information processing of the least squares support vector machine. Combined with the $\mathrm{H}_{2}$ / $\mathrm{H}_{\infty}$ robust control method, simulation results showed that the control system possessed good adaptive ability with regard to sea condition variations. All of the above research used linear feedback control, including the works on other control tasks, for example ship collision avoidance (Szlapczynski, 2011) and fault-tolerant control (Bong, 2015).

A conventional closed-loop system is a linear feedback control scheme; the input signal led to the controller is proportional to the control error. As a result, for smaller control error, the control action produced by the controller may be not enough, on the other hand, for larger control error, the control action may be too strong to eliminate the error (Thomas et al., 2013). Motivated by this argument, a nonlinear feedback control scheme is considered in this research, for example, a cubic function of the error is applied to the controller for a test. However, the control effect is not as much improved as expected in the simulation for an actual ship. When a cubic nonlinear feedback is replaced with a sine function, satisfactory results appear: the control action is obviously reduced while the control quality is kept almost the same as before. In this paper, theoretical analysis and simulation experiments for course keeping manoeuvring of ships using nonlinear feedback control are developed.

\section{CONTROL PROBLEM AND NONLINEAR FEEDBACK TECHNIQUE.}

Consider a course keeping problem for ships, the controlled plant $G$ is taken as the nominal Nomoto model when the controller $K$ is designed using the first order closed loop gain shaping algorithm without considering the nonlinear feedback (Zhang and Wang, 2010; Aarsaether and Moan, 2010). A robust controller for a standard feedback system is solved below under the following three predetermined conditions: the bandwidth frequency of the closed system being $1 / T_{1}\left(1 / T_{1}\right.$ should be the crossover frequency in the strict sense, and is approximately regarded as bandwidth 
frequency for the sake of easy analysis), the largest singular value being unity, and the high frequency asymptote slope being $-20 \mathrm{~dB} / \mathrm{dec}$. The frequency spectrum of the closed-loop system is made equal to the frequency spectrum of a first-order inertial system with the largest singular value approximately one (Zhang and Wang, 2010), i.e.

$$
\frac{1}{T_{1} s+1}=\frac{G K}{1+G K}
$$

The course keeping controller is then solved as

$$
K(s)=\frac{1}{G T_{1} s}
$$

The ship model being a standard Nomoto model is expressed in Equation (2) where $\psi$ is the heading angle, $\delta$ is the rudder angle, $K_{0}$ and $T_{0}$ are the manoeuvrability indices of the ship.

$$
G(s)=\frac{\psi}{\delta}=\frac{K_{0}}{s\left(T_{0} s+1\right)}
$$

To eliminate the steady state error using the closed loop gain shaping algorithm, a minor constant $\varepsilon(0 \cdot 01)$ is added into the denominator of the Nomoto model. Then, Equation (3) is obtained

$$
G(s)=\frac{K_{0}}{T_{0} s^{2}+s+\varepsilon}
$$

Actually, $\varepsilon$ can derive the integral effect for the designed controller and it also reproduces the effect of uncertain constant disturbance upon the closed-loop system. When $\varepsilon$ is too small, the control law may be with the static error or the long setting time. When $\varepsilon$ is too large, the system output tracks the reference signal with the overshooting dynamics. Therefore one should trade off the two terms by selecting the parameter $\varepsilon$ properly.

Thus, substitute Equation (3) into Equation (1), according to closed loop gain shaping algorithm, a linear Proportional-Integral-Differential (PID) controller is obtained

$$
K(s)=\frac{1}{K_{0} T_{1}}+\frac{\varepsilon}{K_{0} T_{1}} \frac{1}{s}+\frac{T_{0}}{K_{0} T_{1}} s
$$

In actual application, we discover that the settling time is relatively long for ships with large time constants such as oil tankers etc. The dynamic performance of course keeping control systems for ships can be improved greatly when a positive constant $\rho$ is added to the proportional part of the control law, Equation (4). Finally, the actual controller is presented in Equation (5). The corresponding theoretical analysis and simulation test are given in reference Zhang (2012).

$$
K(s)=\frac{1}{K_{0} T_{1}}+\rho+\frac{\varepsilon}{K_{0} T_{1}} \frac{1}{s}+\frac{T_{0}}{K_{0} T_{1}} s
$$

A nonlinear feedback system driven by a sine function is shown in Figure 1. Contrary to the standard feedback configuration, $\sin \left(\omega_{1}(r-y)\right)$ where $\omega_{1}$ is the design parameter substituted for $(r-y)$. Note that the block diagram of $\sin \left(\omega_{1}(r-y)\right)$ shown in Figure 1 


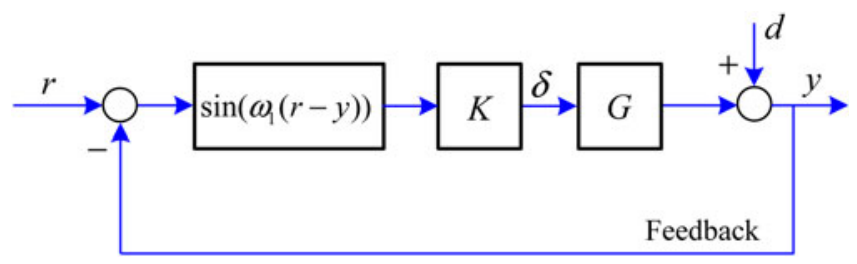

Figure 1. The block diagram of a nonlinear feedback system.

does not conform to its standard graphical representation. How to find a stable $K$ with fine control performance in $\delta=K(r-y)$ is the main work in the previous research no matter whether $K$ is linear or nonlinear, but in this section the main work is how to test the better control performance of the nonlinear feedback control in the form of $\delta=$ $K \sin \left(\omega_{1}(r-y)\right)$ under the same controller $K$.

The effects of nonlinear feedback on the dynamic and static performance are analysed by using $\sin \left(\omega_{1}(r-y)\right) \approx \omega_{1}(r-y)$ when the error is small. The demonstration process is presented in Zhang (2011). In some situations, this assumption may not be tenable when the error is large. The effects of nonlinear feedback driven by sine function to the closed system can be analysed by Taylor series expansion:

$$
\begin{gathered}
\sin \left(\omega_{1}(r-y)\right) \approx \omega_{1}(r-y)-\left(\omega_{1}(r-y)\right)^{3} / 3 ! \\
+\left(\omega_{1}(r-y)\right)^{5} / 5 !-\cdots
\end{gathered}
$$

Let the error $e=r-y$, Equation (6) is simplified up to the third order, then

$$
f(e)=\omega_{1} e-\frac{\omega_{1}^{3} e^{3}}{6}
$$

According to $\mathrm{Hu}$ (2007), if the error input $e$ of Equation (7) is $A \sin \omega_{0} t$, then the output of the nonlinear system in Equation (7) can be approximated by its first order harmonic element, and the equivalent frequency characteristics is the describing function of the nonlinear system.

Let the output of Equation (7) be $f(t)$ under the sine input $A \sin \omega_{0} t$, then its output can be expressed as Equation (8) using its first order harmonic element of the Fourier series (Ciaurri et al., 2010).

$$
f(t)=A_{0}+A_{1} \cos \omega_{0} t+B_{1} \sin \omega_{0} t
$$

where $A_{0}$ is the DC component, $A_{1}, B_{1}$ are the first order harmonic components, and

$$
\left\{\begin{array}{l}
A_{1}=\frac{1}{\pi} \int_{0}^{2 \pi} f(\mathrm{t}) \cos \omega_{0} t \mathrm{~d} \omega_{0} t \\
B_{1}=\frac{1}{\pi} \int_{0}^{2 \pi} f(\mathrm{t}) \sin \omega_{0} t \mathrm{~d} \omega_{0} t \\
A_{0}=\frac{1}{2 \pi} \int_{0}^{2 \pi} f(\mathrm{t}) \mathrm{d} \omega_{0} t
\end{array}\right.
$$

Under the action of sine input signal $e$ in Equation (7), the complex ratio of its first order harmonic element in the steady state output to its input signal is referred to 


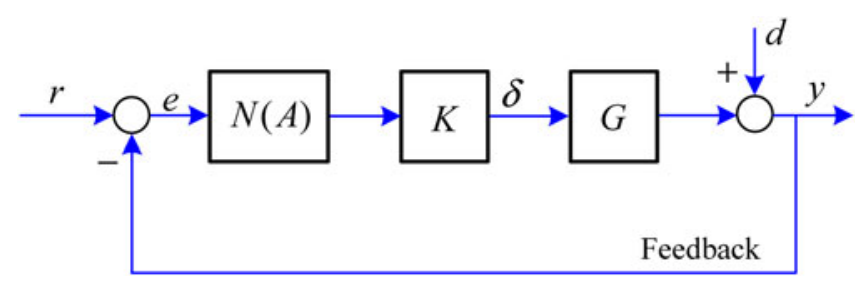

Figure 2. Equivalent block diagram of a nonlinear feedback system.

the describing function which is expressed as $N(\mathrm{~A})$.

$$
N(A)=\frac{B_{1}+\mathrm{j} A_{1}}{A}
$$

Equation (7) is an odd function, thus $A_{0}=0$. When $e=A \sin \omega_{0} t$

$$
f(t)=\omega_{1} A \sin \omega_{0} t-\frac{\omega_{1}^{3} A^{3}}{6} \sin ^{3} \omega_{0} t
$$

Equation (9) is also an odd function of $t$, so $A_{1}=0$. Because of the semi-cyclic symmetry property of $f(t)$, then

$$
\begin{aligned}
& B_{1}=\frac{4}{\pi} \int_{0}^{\pi / 2} f(\mathrm{t}) \sin \omega_{0} t \mathrm{~d} \omega_{0} t \\
& =\frac{4}{\pi}\left[\int_{0}^{\pi / 2} \omega_{1} A \sin ^{2} \omega_{0} t \mathrm{~d} \omega_{0} t-\int_{0}^{\pi / 2} \frac{\omega_{1}^{3} A^{3}}{6} \sin ^{4} \omega_{0} t \mathrm{~d} \omega_{0} t\right] \\
& =\frac{4}{\pi}\left[\omega_{1} A \cdot \frac{\pi}{4}-\frac{\omega_{1}^{3} A^{3}}{6} \cdot \frac{3}{8} \cdot \frac{\pi}{2}\right] \\
& N(\mathrm{~A})=\frac{B_{1}}{A}=\omega_{1}-\frac{\omega_{1}^{3} A^{2}}{8}
\end{aligned}
$$

In the light of the physical meaning of frequency characteristics, the system in Figure 1 is equivalent to the system shown in Figure 2. Effects of the nonlinear feedback driven by sine function can be analysed as follows.

2.1. Effect on the steady state of the closed loop system. Let the reference input be a step signal, its amplitude is $r$, the steady state error to the step input is obtained directly by the final value theorem as given below:

$$
\begin{aligned}
e(\infty) & =\lim _{s \rightarrow 0} s \frac{1}{1+G K N(A)} \frac{r}{s} \\
& =\lim _{s \rightarrow 0} \frac{r}{1+\left(\frac{1}{T_{1} s}+\rho \frac{K_{0}}{s\left(T_{0} s+1\right)}\right) \omega_{1}}=0
\end{aligned}
$$

Therefore the nonlinear feedback driven by sine function has no extra effect to the steady state of the system.

2.2. Effect on the dynamic performance of the closed loop system. Based on the block diagram of the nonlinear feedback system shown in Figure 2, the transfer 
function from the input $r$ (i.e. the setting course $\psi_{r}$ ) to the output $y$ of the system (i.e. the heading angle $\psi$ ) can be obtained as Equation (11).

$$
\frac{y}{r}=\frac{G K N(A)}{1+G K N(A)}
$$

For the ship course keeping problem, wave action is a high frequency disturbance whose frequency spectrum lies in the range of $0.3 \sim 1.25 \mathrm{rad} / \mathrm{s}$. Generally $1 / T_{1}<0.3$ $\mathrm{rad} / \mathrm{s}$ is taken in Equation (10) to shy away from the wave frequency spectrum. Suppose the range of course changing is between $0 \sim 2 \pi \mathrm{rad}$, then $0<N(\mathrm{~A}) \leq \omega_{1}$. The Loop Shaping algorithm of $H_{\infty}$ robust control theory is a type of open-loop gain shaping method (Zhang, 2012). Its key point lies in finding a controller $K$ to make the gains $\underline{\sigma}(G K)$ and $\bar{\sigma}(G K)$ of the open-loop transfer matrix $G K$ satisfy robust performance in the low frequency zone and robust stability in the high frequency zone, i.e. high gain in the low frequency zone and low gain in the high frequency zone. The loop shaping algorithm implements the closed-loop performance of the system through selecting weighting functions to shape the open-loop frequency characteristic curve, and obtains an acceptable performance/robustness trade-off. According to the loop shaping theory, if Equation (11) is compared to the closed loop transfer function $G K /(1+G K)$ of a standard feedback system, the introduction of $N(\mathrm{~A})$ has less effect on the dynamic performance of the system because of the high gain of $G K$ in the low frequency zone and $0<N(\mathrm{~A}) \leq 1$. Thus the proper trade-off between the stabilising performance and robustness can be obtained by selecting the corresponding parameter setting appropriately.

2.3. Effect on the output of the closed loop system. The transfer function from the input $r$ to the output $\delta$ of the controller (i.e. the rudder angle) is

$$
\frac{\delta}{r}=\frac{K N(A)}{1+G K N(A)}
$$

According to $\mathrm{Hu}$ (2007), $K$ has the first order large gain, $G K$ has the second order large gain. Similar analysis as for Equation (11) can proceed showing that the introduction of $N(\mathrm{~A})$ (i.e. the parameter $\omega_{1}$ ) can bring about the benefit of reducing the output of the controller. In addition, the sine function can bring the maximum and minimum values of the error input of the controller within \pm 1 . A similar processing technique can be seen in fuzzy control, neural network and optimising Genetic Algorithms (GA). Certainly, the precondition is to ensure the input of the nonlinear function $\omega_{1} e \in$ $[-\pi / 2, \pi / 2]$ to acquire the preferable effect.

3. NUMERICAL EXAMPLES AND DISCUSSIONS. Taking the Yulong training ship of the Dalian Maritime University as an example, the corresponding ship particulars are: Length between perpendiculars $L=126 \mathrm{~m}$, Beam $B=20 \cdot 8 \mathrm{~m}$, displacement $\nabla=14278.1 \mathrm{~m}^{3}$, draught $D=8.0 \mathrm{~m}$, block coefficient $C_{b}=0.681$, distance from centre of gravity to the origin of $x$ coordinate axis (i.e. the geometric centre) $x_{G}=$ $-3.38 \mathrm{~m}$, ship speed $U_{0}=15 \mathrm{kn}$, rudder area $A_{\delta}=18.8 \mathrm{~m}^{2}$. The manoeuvrability indices of the Nomoto model can be calculated from the above parameters (Zhang, 2012): $K_{0}=0.48 \mathrm{~s}^{-1}, T_{0}=216.58 \mathrm{~s}$. In this simulation, the linear Nomoto model is employed to derive the control law. The parameter settings $\rho=2, T_{1}=0.3 \mathrm{~s}$ are 


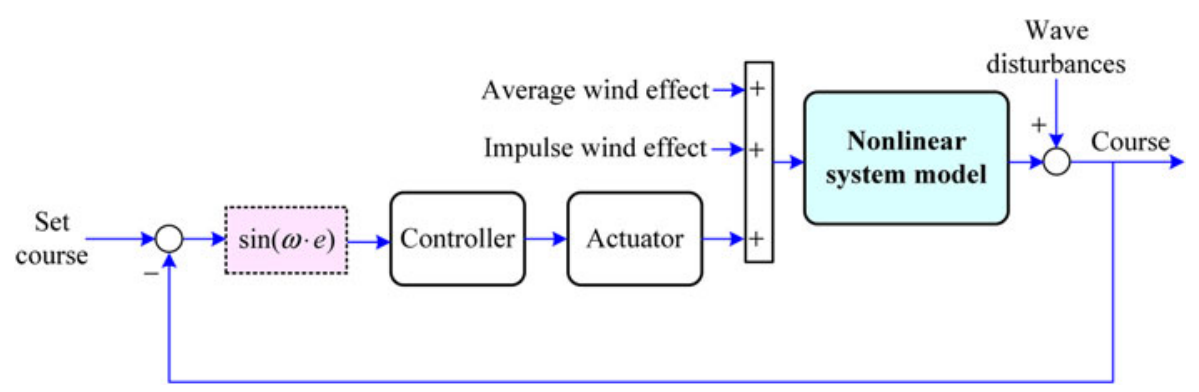

Figure 3. Simulation diagram of Simulink.

employed, which makes the effective working bandwidth frequency of the course keeping controller $1 / 3 \mathrm{rad} / \mathrm{s}$ to avoid overlapping with the wave disturbance range. The nonlinear feedback parameter is selected as $\omega_{1}=0 \cdot 25$. While the nonlinear mathematical model Equations (13) of ship dynamics are considered as the plant to illustrate the effectiveness of the proposed control scheme, which can reflect conditions similar to the real motion of the ship (Fossen, 2011).

$$
\left\{\begin{array}{l}
\left(m+m_{x}\right) \dot{u}-\left(m+m_{y}\right) v r-m x_{G} r^{2}=X_{H}+X_{P}+X_{R} \\
\left(m+m_{y}\right) \dot{v}+\left(m+m_{x}\right) u r+m x_{G} \dot{r}=Y_{H}+Y_{P}+Y_{R} \\
\left(I_{z z}+J_{z z}\right) \dot{r}+m x_{G}(\dot{v}+u r)=N_{H}+N_{P}+N_{R} \\
\dot{\psi}=r \\
\dot{x}_{0}=u \cos \psi-v \sin \psi \\
\dot{y}_{0}=v \cos \psi+u \sin \psi
\end{array}\right.
$$

Where the rudder servo system is also considered in the simulation, the steering engine is modelled as a system with single hydraulic circuit analogue control variable (Zhang, 2012), the maximum rudder ratio is $\pm 5^{\circ} / \mathrm{s}$ and the saturation rudder angle is $\pm 35^{\circ} . u, v$, $r, \psi, x_{0}, y_{0}, n, \delta$ denote linear and angular velocities, heading, positions, propeller rotational speed, rudder angle (expressed in rad) respectively; $m$ and $I_{z z}$ are the ship's mass and mass moment of inertia, respectively. $m_{x}, m_{y}, J_{z z}$ are added mass and added moment of inertia. $X_{H}, Y_{H}, N_{H}, X_{P}, Y_{P}, N_{P}, X_{R}, Y_{R}, N_{R}$ are hydrodynamic forces and moments acting on hull, propeller and rudder, respectively. For more details about the mathematical model, please refer to the references (Jia and Yang, 1999; Fossen, 2011).

When the ship navigates at sea, the sway motion and heading deviation are caused mainly by marine environmental disturbances. In the simulation, one considers sea wind and irregular wind-generated waves. These are simulated by fusing the physicalbased mathematical model. The wind speed (Beaufort No.7) $V_{\text {wind }}=14.25 \mathrm{~m} / \mathrm{s}$, wind direction $\psi_{\text {wind }}=85^{\circ}$. The Joint North Sea Wave Project (JONSWAP) spectrum is adapted to produce the corresponding wind-related waves, which has been defined as an International Towing Tank Conference (ITTC) standard.

The simulation diagram implemented in Simulink is shown in Figure 3, the setting course is $50^{\circ}$. To provide quantification, three popular performance specifications, Equation (14) are employed to evaluate the corresponding algorithm. That is Mean Absolute Error (MAE), Mean Control Input (MCI) and the Total Variation (TV) of the control. MAE is used to measure the performance of the system response, and 

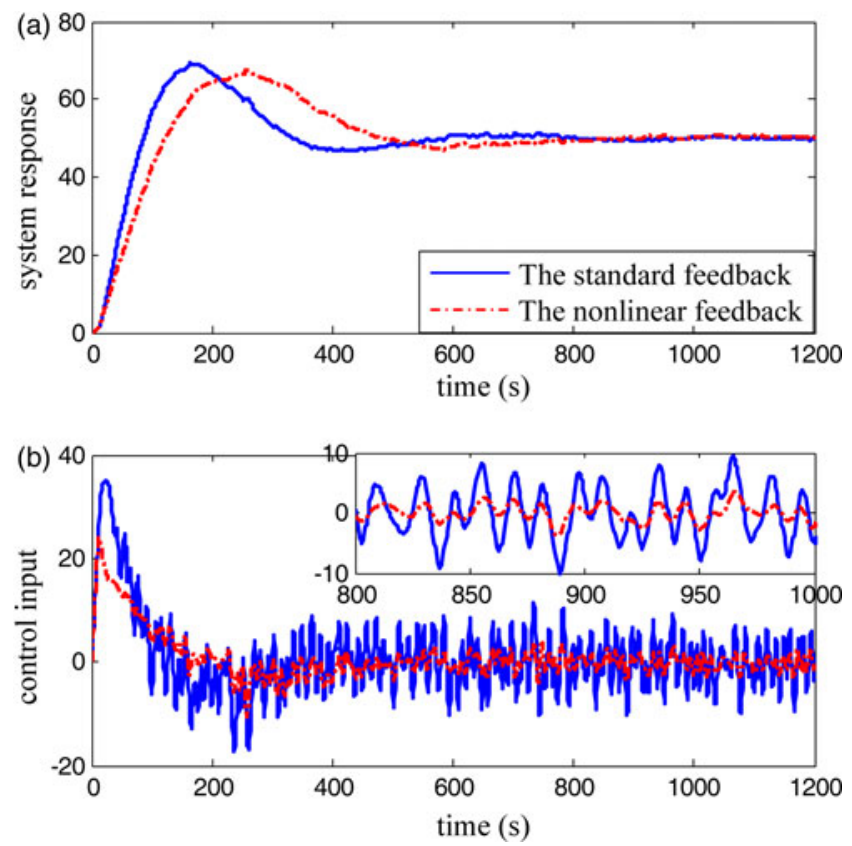

Figure 4. Simulation results for the nominal system: (a) System response, (b) Control input.

MCI and TV measure properties of energy consumption and smoothness. As to the nominal plant, the simulation results are presented in Figure 4. It is noted that the control effect of the nonlinear feedback driven by sine function is almost the same as that in the standard feedback (with the same maximum overshoot and settling time) while the initial maximum rudder angle is reduced to $24.02^{\circ}$ from the original $34.96^{\circ}$ with the drop of $31.2 \%$. There is a $41.4 \%$ drop in the average rudder angle which is decreased on average to $0.0826^{\circ}$ from $0 \cdot 1409^{\circ}$. In order to illustrate the robust performance of the proposed algorithm, the perturbed plant is considered with the speed variation $-50 \%$. The corresponding response dynamics become worse than that of the nominal system. Figure 5 presents the simulation result for the perturbed condition. It is clear to note that saturation is generated in the initial stage using the standard feedback scheme but not under the nonlinear feedback scheme. The other performance indices of the nonlinear feedback scheme are still superior to that of the standard one. In Figures 4(b) and 5(b), the subfigure is a local zoom of the plot, which is to show the control dynamics clearly.

$$
\begin{aligned}
& \text { MAE }=\frac{1}{t_{\infty}-t_{0}} \int_{t_{0}}^{t_{\infty}}|r(t)-y(t)| \mathrm{d} t, \\
& \mathrm{MCI}=\frac{1}{t_{\infty}-t_{0}} \int_{t_{0}}^{t_{\infty}} u(t) \mathrm{d} t, \\
& \mathrm{TV}=\frac{1}{t_{\infty}-t_{0}} \int_{t_{0}}^{t_{\infty}}|u(t+1)-u(t)| \mathrm{d} t
\end{aligned}
$$



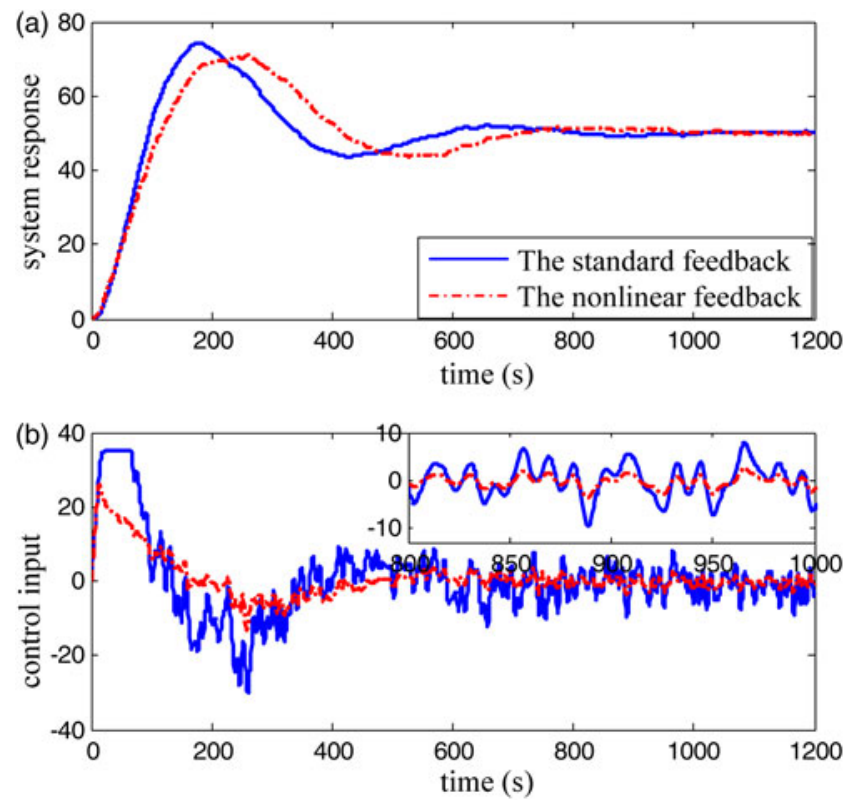

Figure 5. Simulation results for the perturbed system: (a) System response, (b) Control input.

Table 1. Quantitative comparison of the simulation results.

\begin{tabular}{llllr}
\hline & Plant & MAE & MCI & TV \\
\hline The standard feedback & Nominal & 0.09753 & -0.04320 & 0.03281 \\
The nonlinear feedback & & 0.08079 & -0.04347 & 0.01338 \\
The standard feedback & Perturbed & 0.11655 & -0.03889 & 0.03085 \\
The nonlinear feedback & & 0.09208 & -0.03967 & 0.01337 \\
\hline
\end{tabular}

Table 1 gives a quantitative comparison of the simulation results, and this verifies the effectiveness of the nonlinear feedback algorithm. The performance specifications of MAE and MCI are approximated, the smoothness index of the control input is obviously lower than that of the standard feedback method. Under the circumstance of heavy sea state, steering with large rudder angles can increase the amplitude of roll and thus increase the probability of cargo damage and decrease the comfort index of seafarers as well as the safety coefficient of the ship. Therefore reducing the amplitude of rudder angle means that the ship will navigate more safely in addition to being more energy efficient.

In addition, Figure 6 gives the comparisons of the modulating functions $N(e)=e$, $N(e)=\omega_{1} e$ and $N(e)=\sin \left(\omega_{1} e\right)$. As shown in Figure 6, it can be concluded that: the control performance of the nonlinear feedback $N(e)=\sin \left(\omega_{1} e\right)$ is equivalent to that of the linear feedback with an extra constant gain $\omega_{1}$ when the error $e=r-y$ is small; the performance of the nonlinear feedback is superior to that of the linear feedback with an extra constant gain $\omega_{1}$ when the error $e$ is medium; the nonlinear feedback technique cannot work effectively when the error $e$ is too large. It is a very 


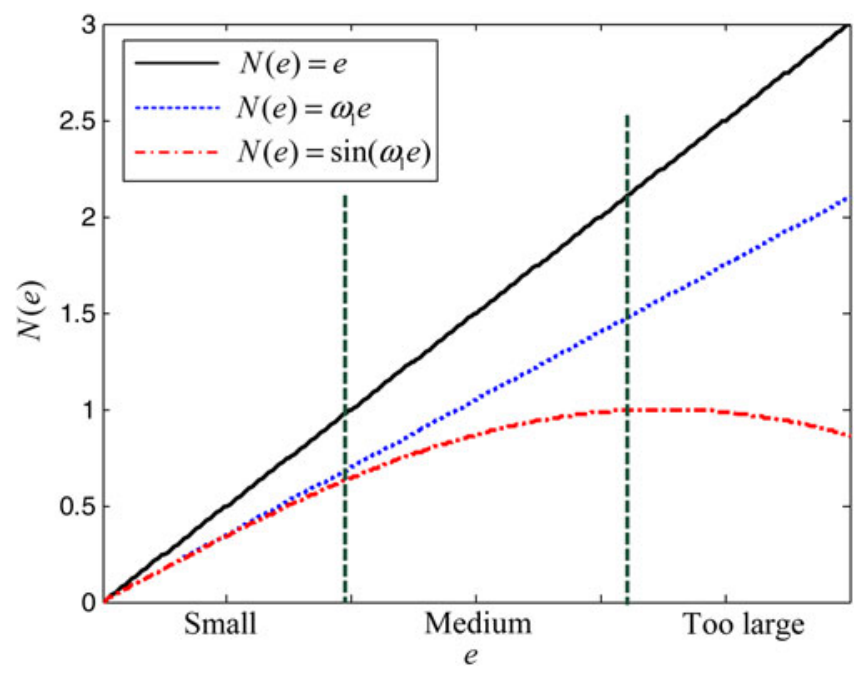

Figure 6. Comparison of the modulating functions (schematic diagram): $N(e)=e, N(e)=\omega_{1} e$ and $N(e)=\sin \left(\omega_{1} e\right)$.

important conclusion that the improvement of control performance is at the cost of the reduction of the system robustness for both schemes by further simulation experiments.

4. CONCLUSION. In this paper a novel idea is presented, that the control error between the reference input and the output is modulated by a sine function and then is fed back to the controller instead of the original direct error, the former is essentially the so-called nonlinear feedback. Nonlinear feedback control can achieve the same control effect with minor control action under the unchanged control law. Taking ship Yulong as an example, simulation results are given using the nonlinear feedback controller driven by the sine function of course error when the set course is $50^{\circ}$ under the wind scale of Beaufort No.7. The rise time is $128 \mathrm{~s}$, the maximum initial rudder angle is reduced to $24.02^{\circ}$ from $34.96^{\circ}$ with $31 \cdot 2 \%$ drop, the average rudder angle is decreased to $0.0826^{\circ}$ from $0 \cdot 1409^{\circ}$ with $41 \cdot 4 \%$ drop, while the control effect is almost the same as that in the linear feedback control. The algorithm has the advantages of energy saving and safety in navigation. The same conclusion can be drawn when the nonlinear feedback is used in some other examples. Hence the algorithm has some universality. However, prudent use needs to be made of the nonlinear feedback technique when the set value is too large.

\section{ACKNOWLEDGMENT}

This work was supported by the National Natural Science Foundation of China (Grant No.51109020) and the Fundamental Research Funds for the Central University (Grant No.2011QN093 and 3132014302). The authors would like to thank Jia Xinle for his insightful remarks on this note, and the anonymous reviewers for their valuable comments to improve the quality of this paper. 


\section{REFERENCES}

Aarsaether, K.G. and Moan, T. (2010). Adding the human element to ship manoeuvring simulations. The Journal of Navigation, 63, 695-716.

Bian, X., Wang, Y., Zhang, X. and Xia, G. (2009). Research on simulation of course control for large ship with wave disturbances in different see conditions. MTS/IEEE Biloxi-Marine Technology for Our Future: Global and Local Challenges, 1-5.

Bong, S.P. (2015). Neural network-based fault-tolerant control of underactuated surface vessels. Mathematical Problems in Engineering, Article ID 903759, in press.

Ciaurri, O., Perez, M., Reyes, J.M. and Varona, J.L. (2010). Mean convergence of Fourier-Dunki series. Journal of Mathematical Analysis and Applications, 372(2), 470-485.

Du, J., Guo, C., Yu, S. and Zhao, Y. (2007). Adaptive autopilot design of time-varying uncertain ships with completely unknown control coefficient. IEEE Journal of Oceanic Engineering, 32(2), 346-352.

Fossen, T.I. (2011). Handbook of Marine Craft Hydrodynamics and Motion Control. John Wiley \& Sons, New York.

Ho, W.H., Hsieh, C.H. and Chou, J.H. (2010). Optimal course handing control for nonlinear ship maneuvering systems, International Journal of Innovative Computing, Information, and Control, 10(6), 4379-4388.

$\mathrm{Hu}$, S. (2007). Automatic control principle (Version No.5). Science Press, Beijing.

Jia, X. and Yang, Y. (1999). Ship Motion Mathematic Model (the mechanism modeling and the identification modelling), Dalian Maritime University Press.

Johansen, T.A. and Fossen, T.I. (2013). Control allocation-a survey. Automatica, 49(5), 1087-1103.

Krstic, M., Kanellakopoulos, I. and Kokotovic, P. (1995). Nonlinear and Adaptive Control Design. Wiley, New York.

Liu, S., Song, J. and Li, G. (2010). Robust LSSVM control for ship course-keeping system. Control and Decision, 25(4) 551-556.

Satpati, B., Bandyopadhyay, I., Koley, C. and Ojha, S.K. (2008). Robust controller design for course changing/course keeping control of a ship using PSO enabled automated quantitative feedback theory. TENCON 2008 IEEE Region 10 Conference, 1-6.

Szlapczynski, R. (2011). Evolutionary sets of safe ship trajectories: a new approach to collision avoidance. The Journal of Navigation, 64, 169-181.

Thomas, B., Sebastian, H. and Knut, G. (2013). Nonlinear model predictive control of a magnetic levitation system. Control Engineering Practice, 21(9), 1250-1258.

Velasco, F.J., Herrero, E.R., Lopez, E. and Moyano, E. (2013). Identification for a heading autopilot of an autonomous In-Scale Fast Ferry. IEEE Journal of Oceanic Engineering, 38(2), 263-274.

Zhang, G. and Zhang, X. (2012). Stability analysis and design of integrating unstable delay processes using the mirror mapping technique. Journal of Process Control, 24(7), 1038-1045.

Zhang, X. (2011). Control Algorithm of Autopilot Driven by Sine Function of Course Deviation. Navigation of China, 34(1), 1-5.

Zhang, X. (2012). Concise Robust Control for Marine Ships. Science Press, Beijing.

Zhang, X. and Wang, X. (2010). Concise nonlinear robust control of course keeping for ships. ICIC Express Letters, 4(4), 1263-1268. 